\title{
High-resolution optical modeling of the Thirty Meter Telescope for systematic performance trades
}

Carl Nissly, Byoung-Joon Seo, Mitchell Troy, George Angeli, John Angione, et al.

Carl Nissly, Byoung-Joon Seo, Mitchell Troy, George Angeli, John Angione, lan Crossfield, Brent Ellerbroek, Luc Gilles, Norbert Sigrist, "High-resolution optical modeling of the Thirty Meter Telescope for systematic performance trades," Proc. SPIE 7017, Modeling, Systems Engineering, and Project Management for Astronomy III, 70170 (9 July 2008); doi: 10.1117/12.788596

Event: SPIE Astronomical Telescopes + Instrumentation, 2008, Marseille, France 


\title{
High-resolution optical modeling of the Thirty Meter Telescope for systematic performance trades
}

\author{
Carl Nissly ${ }^{a, c}$, Byoung-Joon Seo ${ }^{a, c}$, Mitchell Troy ${ }^{a, c}$, \\ George Angeli ${ }^{b, c}$, John Angione ${ }^{a, c}$, Ian Crossfield ${ }^{d}$, \\ Brent Ellerbroek $^{b, c}$, Luc Gilles $^{b, c}$, and Norbert Sigrist ${ }^{a, c}$ \\ ${ }^{a}$ Jet Propulsion Laboratory, Pasadena, CA. 91109 \\ ${ }^{b}$ Thirty Meter Telescope Observatory, Pasadena, CA. 91125 \\ ${ }^{c}$ California Institute of Technology, Pasadena, CA. 91125 \\ ${ }^{d}$ University of California, Los Angeles, CA. 90095
}

\begin{abstract}
We consider high-resolution optical modeling of the Thirty Meter Telescope for the purpose of error budget and instrumentation trades utilizing the Modeling and Analysis for Controlled Optical Systems tool. Using this ray-trace and diffraction model we have simulated the TMT optical errors related to multiple effects including segment alignment and phasing, segment surface figures, temperature, and gravity. We have then modeled the effects of each TMT optical error in terms of the Point Source Sensitivity (a multiplicative image plane metric) for a seeing limited case and an adaptive optics corrected case (for the NFIRAOS). This modeling provides the information necessary to rapidly conduct design trades with respect to the planned telescope instrumentation and to optimize the telescope error budget.
\end{abstract}

Keywords: Optical Modeling, MACOS, Point Source Sensitivity, NFIRAOS, Thirty Meter Telescope

\section{INTRODUCTION}

The Thirty Meter Telescope (TMT) design is a highly segmented mirror telescope with an equivalent aperture of 30 meters. The f/1 primary mirror is made up of 492 hexagonal segments, each with a $1.44 \mathrm{~m}$ corner-to-corner width. The Ritchey-Chretien telescope system is designed to have an f/15 final focus with a 20 arc-minute Field of View, and a wavelength coverage from 0.31 to 28 microns. ${ }^{1}$

The JPL developed Modeling and Analysis for Controlled Optical Systems (MACOS) software ${ }^{2}$ is a tool for optical systems analysis developed at the Jet Propulsion Laboratory. It has been used on projects such as JWST, SIM, TPF, SST, and HST for optical and integrated modeling purposes. The MACOS tool has capabilities, which range from modeling complex surface geometries, atmospheric effects, and detector simulations using both sequential and non-sequential ray tracing and diffraction calculation. MACOS has the desirable capability to run through a Matlab (The MathWorks, Inc.) interface, which provides for a vast range of use including high-resolution surface modeling, optical sensitivity calculations, and wavefront sensing and control simulations.

Using MACOS we have developed a high-resolution optical model of TMT for system engineering performance and sensitivity analyses. The MACOS model provides a means to rapidly model the wavefront quality of the telescope and has been used to simulate mirror segment surface figure control, alignment and phasing methods, and to validate the optical performance error budget.

The normalized Point Source Sensitivity (PSS) is a multiplicative image performance metric developed by the TMT systems engineering group published in Ref. Seo-2008. The PSS is defined in Eq. 1.

$$
P S S_{\text {Normalized }}=\frac{\int_{\infty}\left|P S F_{\text {Error }+ \text { Atmosphere }}(\vec{\theta})\right|^{2} d \vec{\theta}}{\int_{\infty}\left|P S F_{\text {Atmosphere }}(\vec{\theta})\right|^{2} d \vec{\theta}}
$$

Here we calculate the integral of the PSF squared for the aberrated telescope error convolved with the atmosphere $\left(P S F_{\text {Error+Atmosphere }}\right)$ and normalize it to the perfect telescope convolved with the atmosphere $\left(P S F_{\text {Atmosphere }}\right)$. The normalized PSS, which in this paper we denote as PSS, is a measure of the science loss for seeing limited

Modeling, Systems Engineering, and Project Management for Astronomy III, edited by George Z. Angeli,

Martin J. Cullum, Proc. of SPIE Vol. 7017, 70170U, (2008) · 0277-786X/08/\$18 · doi: 10.1117/12.788596 
instruments. TMT has a requirement that the telescope should not introduce more than a $10 \%$ loss of science, which suggests that TMT would like to achieve a PSS value on the order of 0.9. The normalized PSS of individual error terms can be multiplied together to calculate an estimate of the total normalized PSS. ${ }^{3}$

In Section 2 of this paper we discuss the details of the modeling tools and assumptions. Section 3 describes each of the modeled error terms. Section 4 presents the simulation results as related to seeing limited and AO instruments.

\section{MODELING TOOL}

\subsection{MACOS Model}

\subsubsection{Prescription Generator}

In order to produce an optical model of TMT with such a large number of primary segments, it was necessary to create a tool that can automatically and rapidly generate the optical prescription for MACOS to read. This tool then allows for easy model modification, whether for the purpose of model debugging or for a quick adjustment to the fundamental optical design. This capability was essential early on in the modeling process as TMT system parameters were often modified. The MACOS prescription contains rotation points, curvatures, conics, and local coordinate frames for each of the 492 segments as well as for the secondary and tertiary mirrors. The volume of this data shows the clear necessity for such a tool.

\subsubsection{Segmented Model}

The MACOS model gives the ability to model the highly segmented TMT primary mirror within reasonable simulation times. The optical ray-trace is conducted using a sequential surface methodology. The light source is setup to match the exact hexagonal geometry of the primary mirror. A regularly sampled grid of rays is then traced through the reflective surfaces of the system. This method allows for a significant computation speed advantage over the non-sequential alternative because each ray is traced to the mirror segment that its source segment maps to. MACOS traces 2.7 million rays to form a 2048x2048 pixel exit pupil.Fig. 1 shows a sample Optical Path Difference (OPD) map for the segmented model calculated at the exit pupil. MACOS can then perturb these mirror segments as rigid-body translations and rotations or as surface shape deformations to model systematic telescope errors.

\subsubsection{Optical Model Assumptions}

The following is a list of assumptions and approximations that we have made during the implementation of our optical TMT model. First we assume that the primary mirror is made up of a regular hexagon grid. This means that our primary segments each have the same regular hexagonal shape in projection. Our OPD map exit pupil sampling is $1 / 64 \mathrm{~m}$ per pixel. Also each mirror surface deformation is approximated using a regularly sampled grid. The primary mirror segments each use a 99x99 pixel grid, the secondary a 401x401 pixel grid, and the tertiary a $461 \times 461$ pixel grid. These pixel resolution values were selected so that we can accurately model the errors terms described in Section 3. We currently ignore the effects of the secondary supports for the seeing limited simulations, it is currently included for the adaptive optics corrected case.

Our default simulation parameters include a wavelength of $500 \mathrm{~nm}$ and atmospheric $r_{0}$ equal to $200 \mathrm{~mm}$ at the zenith. Currently we consider on-axis performance for the telescope errors as a simplification with the intent to expand the maturity of our model to include off-axis measurements in the future. Last, the segment aberrations assume a flat primary mirror approximation with respect to gravity. This means that the M1 segment level errors related to gravity do not account for the surface sag, again this is an approximation that we can add maturity to in the future. 


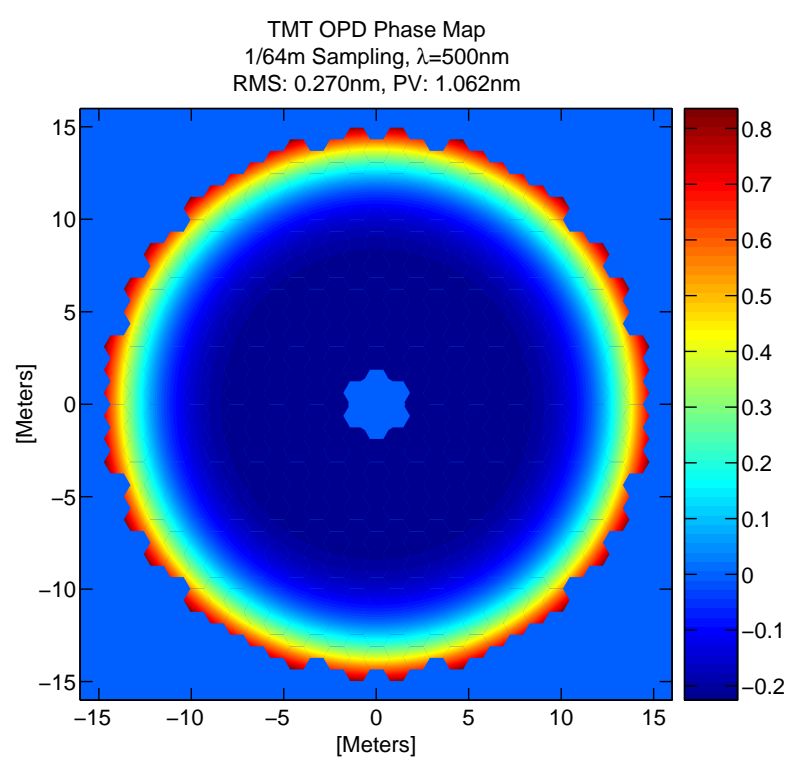

Figure 1. Sample TMT Phase Map, ommiting M2 supports. Note the nominal TMT has $\leq 0.3$ nm of wavefront error

\subsection{Implementation}

\subsubsection{Matlab Executable (MEX)}

MACOS has the capability to run through a Matlab interface using a Matlab EXecutable (MEX) function. This functionality works by using Matlab to define the calling input parameters needed to perturb elements and trace the necessary rays through the system. Matlab then calls a MACOS MEX function, passing these perturbation values and system state parameters to return the outputs of OPD, ray-position spot diagram, and wavefront error back to Matlab as variables. The user then has the extensive calculation power inherent in Matlab to calculate desired optical metrics such as the point-spread function, optical transfer function, point source sensitivity, and encircled energy for the perturbed case.

When active or adaptive surface correction is desired, a simple control loop can be setup for Matlab to correct the mirror surface based upon each looped OPD returned from MACOS. The output of a simple integrating controller and sensitivity model calculates the commands necessary to correct the surface. These new states are returned to the MEX function for the determination of a new system OPD calculation.

\subsubsection{System Error Generation}

We have modeled the optical system errors of TMT using Matlab to calculate surface shape change data and six degree of freedom translations and rotations to simulate the optical telescope errors. We then pass these parameters to MACOS for ray-tracing and OPD calculation at the exit pupil. The Matlab suite of functions for calculating these system perturbations is divided into three main parts, one for each mirror. Each of these functions takes the inputs of telescope angle, delta temperature, and a series of options for running parametric studies and isolating system effects. The outputs of these functions are then the parameter vectors in the MACOS preferred format ready for the OPD calculation. Section 3 describes the generation of each error individually.

\subsubsection{Model Verification}

MACOS has been used for multiple JPL flight and non-flight projects producing results that agree with standard optical ray-trace codes such as Zemax ${ }^{\complement}$ and $\mathrm{CodeV}^{\complement}$. However, we have also validated our TMT MACOS results against a simplified TMT CodeV model and there is excellent agreement. ${ }^{4}$ We have also verified our PSS calculations through a comparison with a colleague, John Pazder from the Herzberg Institute of Astrophysics (HIA), who calculated PSS values for Zernikes over the TMT pupil which showed extremely accurate agreement. 


\subsection{PSS Estimator}

The multiplicative characteristic of the PSS ${ }^{3}$ allows for estimation of the PSS value for combined error cases by calculating the product of its individual parts. We have used this feature to develop a tool for estimating the performance of the telescope for system engineering parametric studies. First we pre-calculate the PSS values for each individual term along evenly spaced sampling of its input (e.g., zenith angle, temperature delta, APS error options, and surface figuring error options). We then have look-up tables for the Matlab interpolation routines to estimate PSS values for each error. These PSS values are estimated individually, with an adjusted zenith $r_{o}$ seeing value, and then multiplied to form a total estimation of the system's performance.

For example, if the user's input is to estimate the PSS at zenith angle 30 degrees and delta temperature of 4 degrees at an $r_{0}$ of $200 \mathrm{~mm}$ at the zenith, Matlab will interpolate a PSS value for each individual error with these inputs and then multiply all of the individual values to form an estimate for each mirror and for the combined telescope. This tool is extremely useful in estimating system performance for a bottom-up performance estimation and also to run Monte-Carlo simulations to estimate thermal and dynamics performance in terms of the point source sensitivity. The figures contained in the seeing limited simulation results Section 4.1 were generated using this tool.

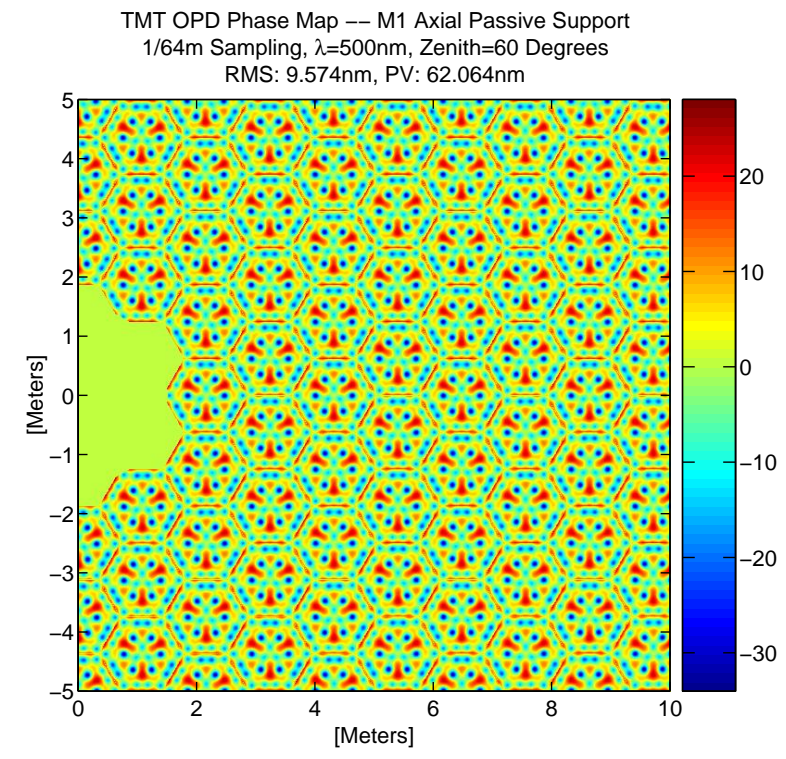

(a) Axial passive support error zoomed in

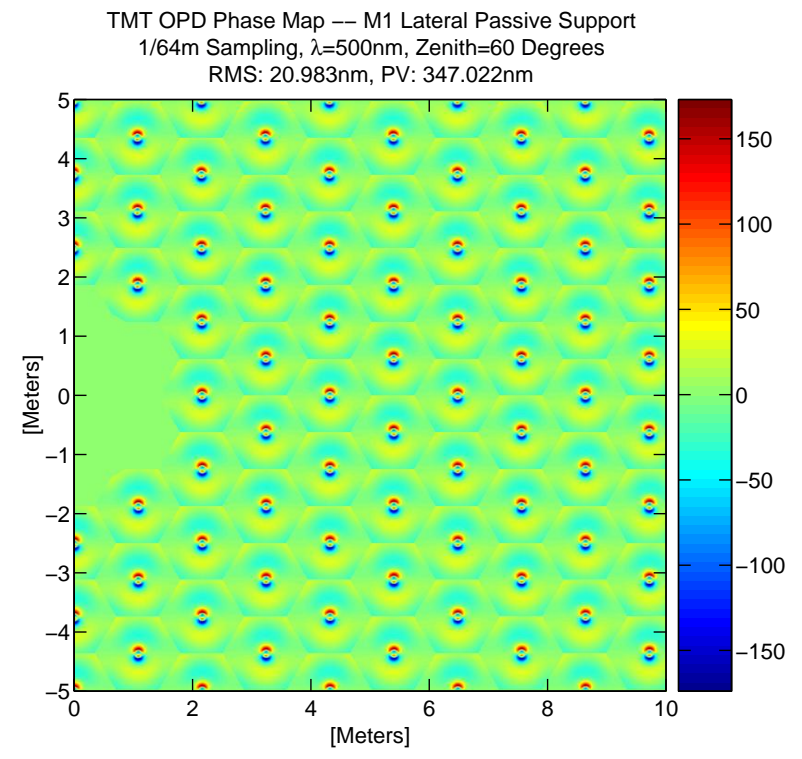

(b) Lateral passive support error zoomed in

Figure 2. M1 Passive Support OPDs, Mirror Case 4

\section{TMT MODELED ERRORS}

\subsection{M1-Primary Errors}

In this section we describe the error terms that we have modeled relating to the primary mirror. We have also modeled error terms related to the secondary and tertiary mirrors; these include passive support, figuring error, and on-axis APS alignment errors. We model these terms with the same methods used for the primary mirror errors described in the following section.

\subsubsection{Segment Passive Support Errors}

The support structure on the back of the primary mirror segments generates a print-through pattern that varies with the changing gravity vectors as a function of zenith pointing angle and is broken into two components, axial 
and lateral. This effect has been minimized by Eric Williams and Vince Stephens at Imtec ${ }^{(c)}$ by optimizing the mirror support pattern, segment material, and segment thickness. The MACOS model has played a valuable part in this optimization process as we implement each of the passive support mirror cases and report RMS wavefront error and point-source sensitivity values. The current baseline uses a radially-slotted central diaphragm design and $45 \mathrm{~mm}$ thick regular Invar segments. Imtec ${ }^{\complement}$ provides us with a regularly sampled grid of surface deformation for both axial and lateral segment shapes. We then scale these maps according to Eq. 2 to simulate the telescope pointing.

$$
\text { Combined }=\text { Lateral } * \sin \left(\theta_{\text {Zenith }}\right)-\text { Axial } *\left(1-\cos \left(\theta_{\text {Zenith }}\right)\right)
$$

The same wavefront error map is applied to all segments with a resolution of $\sim 14 \mathrm{~mm} /$ pixel. This means that we assume that M1 is flat and that we ignore the Segment Support Assembly (SSA) rotation due to segment types and sector ordering. Also note that Eq. 2 assumes that the axial and lateral errors are zero when the telescope if at the zenith. Figure 2(a) shows a sample OPD map of the axial case with an RMS wavefront error of $9.6 \mathrm{~nm}$. Figure 2(b) shows an OPD for the lateral passive support error with an RMS wavefront error of 21.0nm.

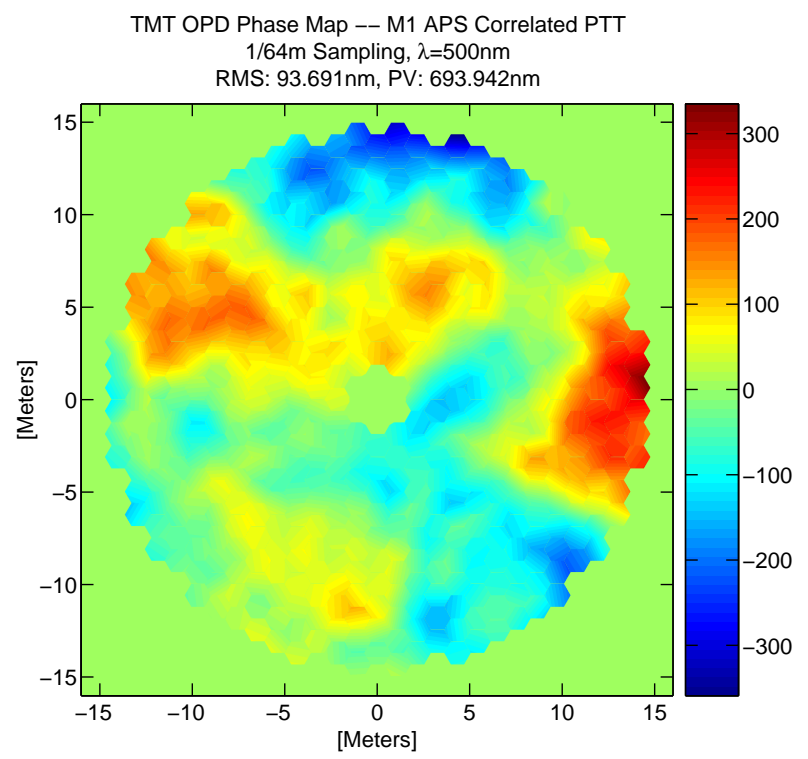

(a) M1 APS correlated piston, tip, and tilt

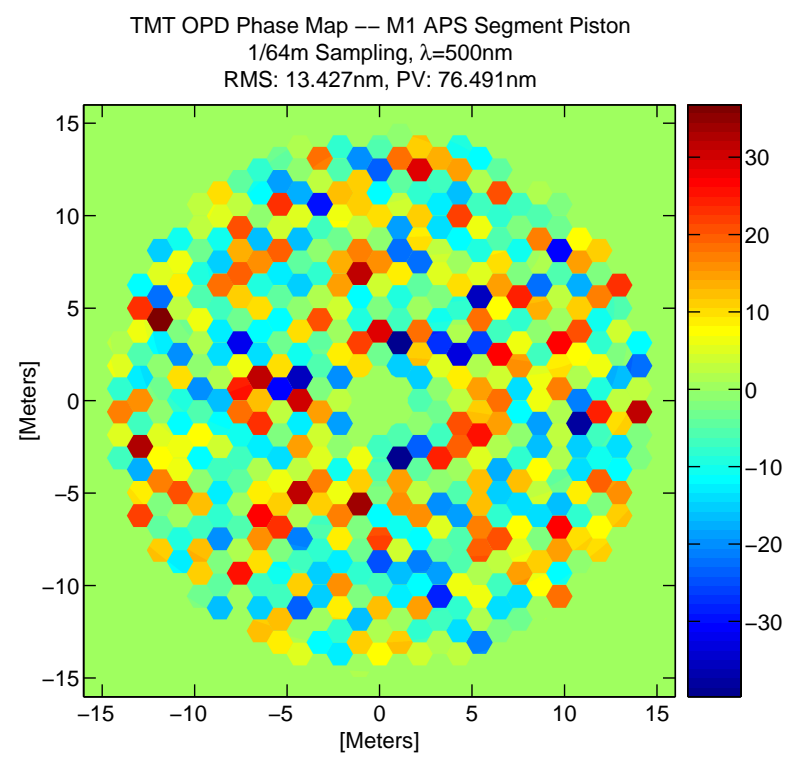

(b) M1 APS random segment piston

Figure 3. Segment piston, tip, and tilt error OPDs

\subsubsection{Segment Piston, Tip, and Tilt Errors}

The Alignment and Phasing System (APS) is responsible for alignment and mirror shape estimation for TMT. For detailed background of the APS system see Ref. Troy-2008..$^{5}$ The largest source of error in the mirror alignment is from the atmosphere. The APS measurement errors are based on data from the Phasing Camera System (PCS $)^{6}$ of the Keck Telescopes scaled for TMT and also a separate MACOS modeling activity as seen in Ref. Seo-2007. ${ }^{7}$

The atmospheric portion of the APS error is modeled over the primary mirror using a correlated piston, tip, and tilt of the segments. We approximate the atmosphere through a Zernike decomposition as proposed by Chanan using an integration time of 600 seconds. $^{5}$ Previous modeling work ${ }^{7}$ has generated a set of Zernike covariance matrices to simulate the $\mathrm{AO}$ correction of this time-averaged atmosphere. Using these covariance terms, we scale the time-averaged Zernike coefficients and pass them to a Matlab routine used to calculate segment rigid-body motion to simulate this corrected atmospheric surface. Fig. 3(a) shows a sample phase map of this atmospheric error with an RMS wavefront error of $93.7 \mathrm{~nm}$. 
The segment phasing error, due to measurement accuracy, is simulated as a random effect with a standard deviation of $6.8 \mathrm{~nm}$ of surface error. ${ }^{6}$ Our routine for implementing this error uses the Z-translation rigid-body segment motion to position the primary mirror segments within this distribution. Fig. 3(b) shows an OPD map of this random segment piston with an RMS wavefront error of $13.4 \mathrm{~nm}$.

\subsubsection{Gravity Clocking and Decenter}

As the telescope moves through its zenith pointing angles, the gravity vector on each of the segments changes significantly and introduces an in-plane clocking and decenter motion of the individual segment's mirror cell. Scott Roberts of HIA has provided the segment clocking and decenter information for three telescope angles. Fig. 4(a) shows a sample vector-field plot of this segment motion at a zenith angle of 65 degrees. This FEM data models the mirror cell in-plane motion and does not currently include the SSA deflection. Since the APS calibration angle is 30 degrees, we assume that the error is corrected by the warping harnesses at this angle, which provides a normalization for the segment clocking and decenters. We then linearly interpolate between the angles for our segment errors due to gravity. Using the telescope zenith angle as an input, we calculate the three degree-of-freedom rigid-body in-plane motion to pass into MACOS to simulate this gravity effect on the primary mirror segments. Fig. 4(b) shows a phase map of this gravity error with an RMS wavefront error of $5.0 \mathrm{~nm}$ at a 60 degree zenith pointing.

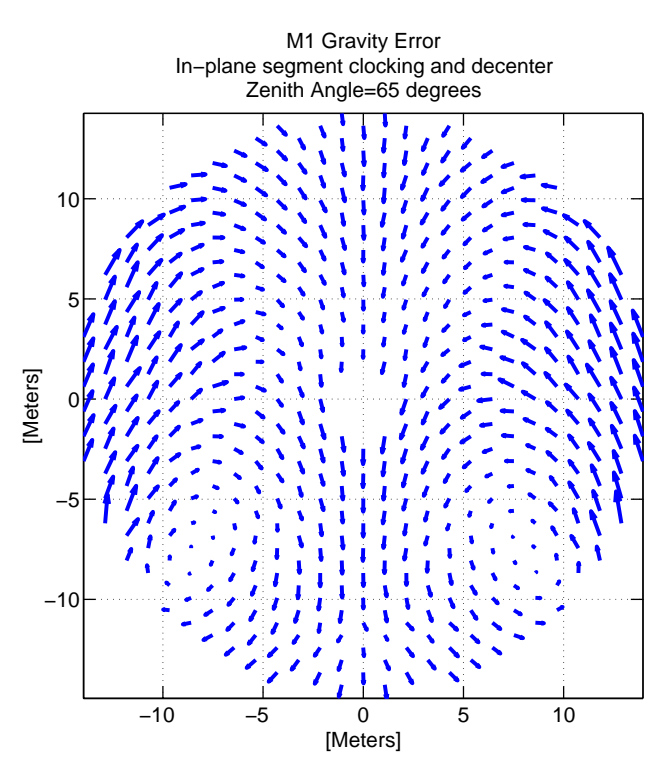

(a) In-plane segment motion vector field

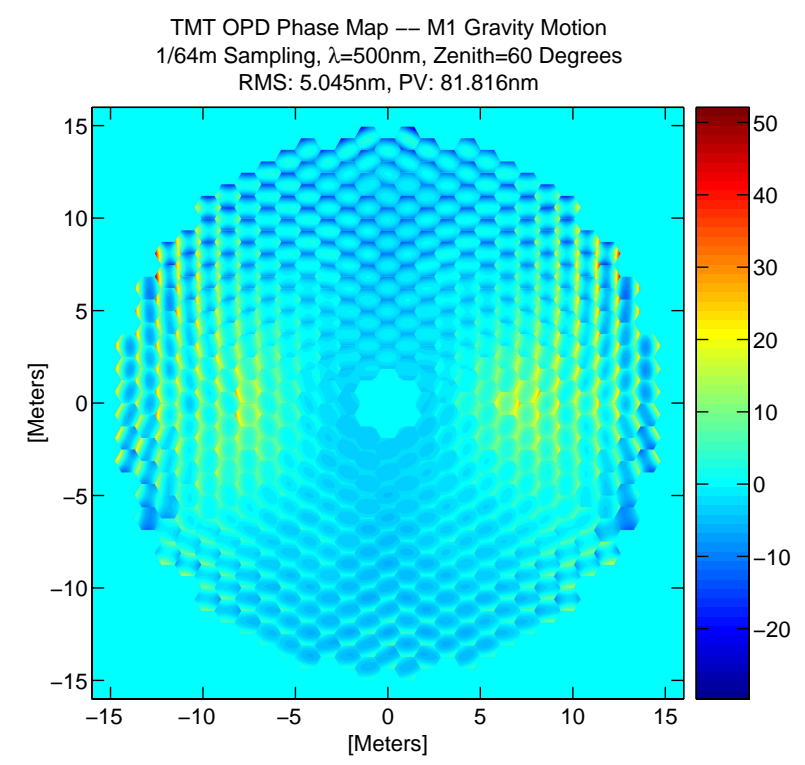

(b) In-plane gravity error OPD

Figure 4. M1 gravity decenter and clocking

\subsubsection{Segment Figuring Error with Warping Harness Correction}

The M1 segment figuring error and surface shape correction is perhaps the most complex of the telescope errors that we have modeled to date. First, the TMT telescope department specifies a structure function to the mirror polisher which defines the required accuracy of polishing. The structure function is the tip/tilt removed atmospheric structure function shown in Eq. 3, where $\mathrm{B}=3.1623[\mathrm{~nm}], \mathrm{d}=1.432[\mathrm{~m}]$, and $r_{0}=1[\mathrm{~m}]$ for the primary mirror.

$$
\begin{gathered}
D(x)=A\left[10.6\left(\frac{x}{d}\right)^{5 / 3}-13.75\left(\frac{x}{d}\right)^{2}+3.42\left(\frac{x}{d}\right)^{3}\right]+2 B^{2} \\
A=\left(\frac{\lambda}{2 \pi}\right)^{2} \times\left(\frac{D}{r_{0}}\right)^{5 / 3}
\end{gathered}
$$




$$
\begin{gathered}
B=\text { High order surface roughness } \\
x=\text { Separation between point pairs } \\
d=\text { Diameter of beam footprint } \\
D=\text { Diameter of Telescope } \\
r_{0}=\text { Quasi-Fried's parameter } \\
\lambda=\text { Wavelength }
\end{gathered}
$$

This structure function has a white-noise term (B) that we ignore, making $\mathrm{B}=0$. This is because to accurately model a white surface noise would require significantly higher spatial resolution than we currently have. With a surface grid sampling of 99x99, we have a spatial resolution of $\sim 12.6 \mathrm{~mm}$. The mirror specification also allows for some additional low order aberrations. APS then measures the shape of the primary mirror segments with some estimated precision. ${ }^{6}$ Next, a set of warping harness forces attempt to correct the surface shape of the segments based on this APS estimate. Their performance is specified by a set of correction factors per Zernike as estimated by Keck segment warping harness performance. This warping harness correction induces an additional surface deformation of the mirror shape that has a high spatial frequency. The input for this surface correction error comes as deformation maps from Eric Williams at Imtec ${ }^{\complement}$, which we scale according to the magnitude of the warping harness correction.

Table 1 shows the method that we use to create the segment shape figuring error term as a function of Noll Zernike order. The M1 structure function is broken down into Zernike coefficients shown in column 2. Column 3 shows the additional allowance terms that are RSSed into each Zernike order. A reduction factor is then applied according to the design of the segment warping harnesses, shown in column 4 . The next column shows the result of this calculation. Column 7 then shows the RSS combination of this segment surface error with the APS shape measurement error that is listed in column 6. These total RMS surface errors are then fed into a Zernike surface composition routine. Finally, a surface grid of the warping harness correction residual is added to this surface. A gridded surface deformation array has then been generated for MACOS to model the mirror segment shape for each of the 492 mirror segments. Fig. 5 shows a sample phase map of this primary mirror shape error with an RMS wavefront error of $25.1 \mathrm{~nm}$. This method assumes that there is no drift in the segment shapes and warping harness positions based upon the monthly measurements taken by the alignment and phasing system. This method also requires that we make the assumption that the mirror segments can be polished to match the atmosphere specified by the structure function.

Table 1. Calculation of M1 figuring error with warping harness, APS, and additional allowance terms per Zernike

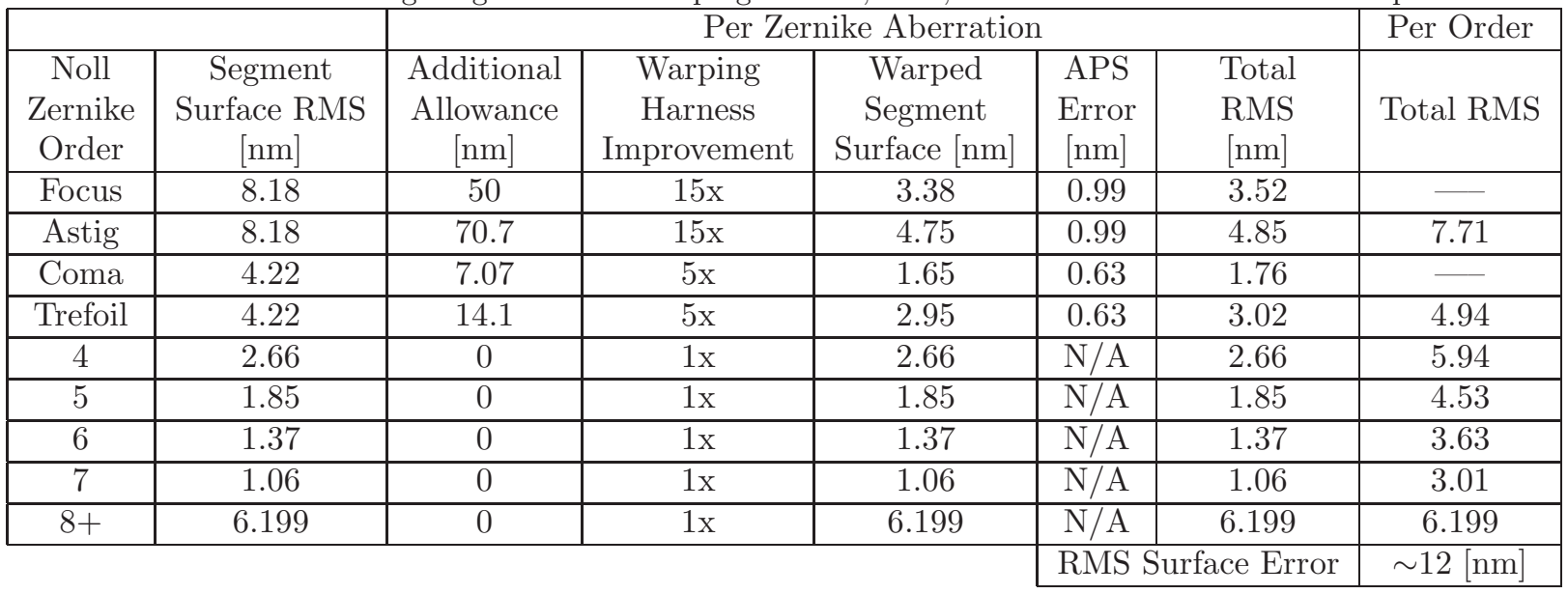




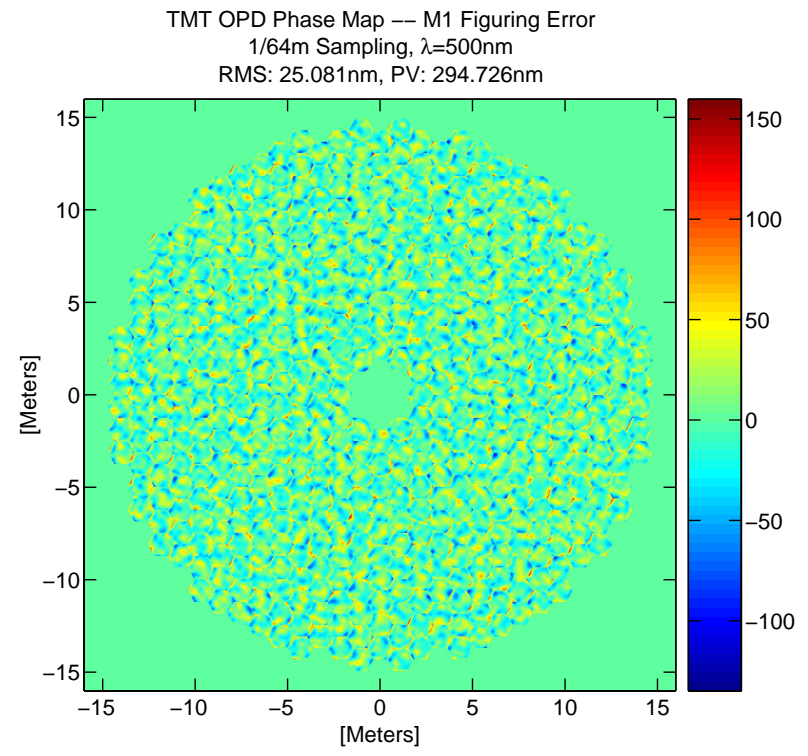

(a)

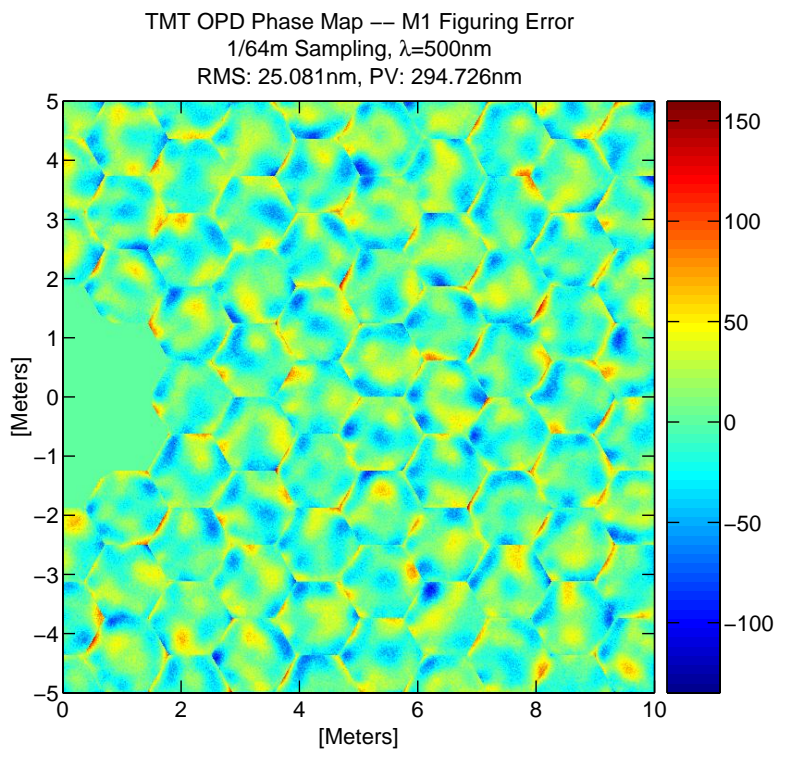

(b)

Figure 5. M1 Figuring Error with Warping Harness Correction OPD

\subsubsection{Thermal Disturbances}

The primary mirror thermal disturbance errors are divided into two components: segment distortion and segment clocking. The temperature delta is the temperature difference relative to the summit temperature at the time of APS calibration. For modeling and error budget validation we used a $\Delta \mathrm{T}$ of 4 degrees as a result of site testing and mean seeing conditions. ${ }^{8}$

The segments distort as a function of temperature at a rate which corresponds to the segment material and the passive support design. The data input for this segment distortion is a regularly sampled grid across the segment of Z-direction surface deformation and is provided by Eric Williams and Vince Stephens of Imtec ${ }^{\complement}$ with each of the passive support designs. The current baseline surface error is $2.4 \mathrm{~nm} /{ }^{\circ} \mathrm{C}$. This segment distortion is simulated using the gridded surface shape perturbation parameter. The next modeling iteration for this thermal distortion error will include local deformations from segment thermal controllers to include the SSA thermal effects. We model this thermal segment distortion with the assumption that the segments have been figured and tested at the summit temperature. Fig. 6(a) shows a sample phase map for a $\Delta \mathrm{T}$ of 4 degrees with an RMS wavefront error of $26.5 \mathrm{~nm}$.

The segment clocking as a function of temperature is caused by a CTE mismatch between the mirror segment and primary mirror support materials. The segments are mounted in six pie slice primary mirror sectors corresponding to the six-fold symmetry of the primary mirror. All of the segments within a sector clock together in the same direction according to an angle per ${ }^{o} \mathrm{C}$. The segments in the adjacent sector then clock in the opposite direction. This segment clocking coefficient is currently $6.8 \mu \mathrm{rad} /{ }^{\circ} \mathrm{C}$. We simulate this segment clocking using the rotation angle rigid-body parameter. Fig. 6(b) shows a sample phase map for $\Delta \mathrm{T}$ of 4 degrees with an RMS wavefront error of $2.4 \mathrm{~nm}$. 


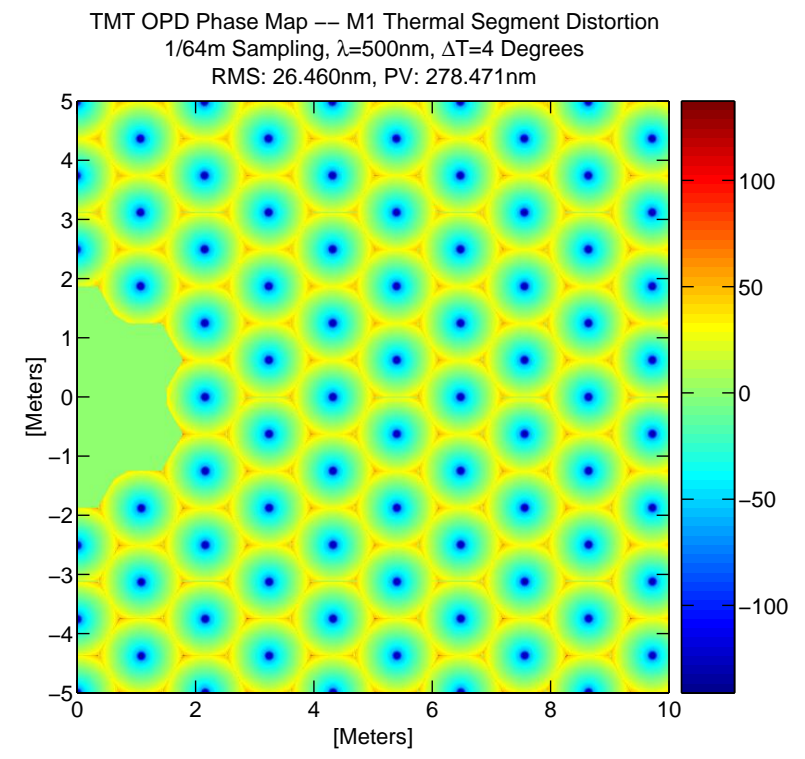

(a) M1 thermal segment distortion zoomed in

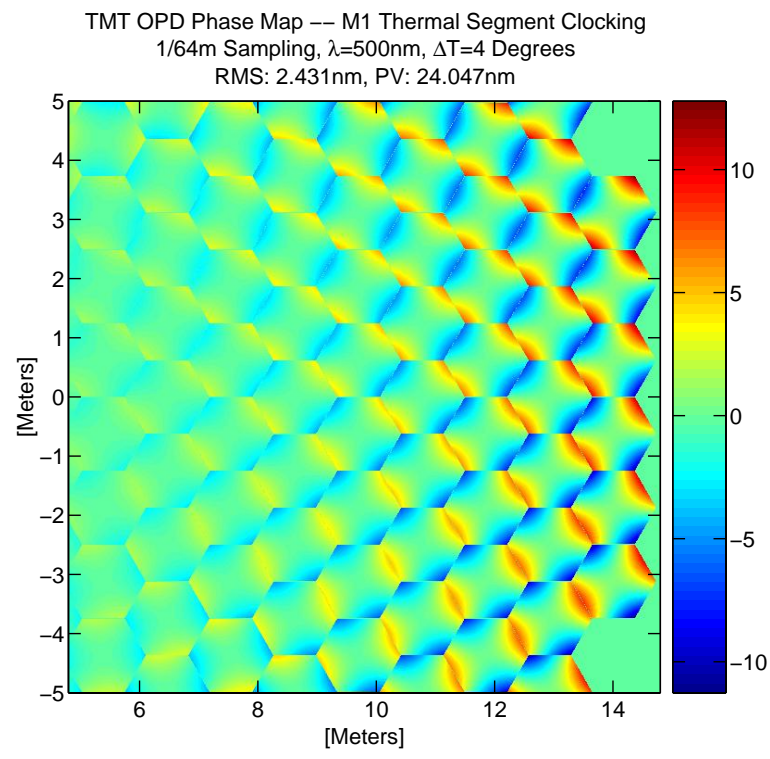

(b) M1 thermal segment clocking zoomed in

Figure 6. M1 thermal error OPDs

\section{SIMULATION RESULTS}

Due to TMT's large range of instruments and applications there are three categories of optical performance estimates that are useful. For seeing limited results, this MACOS modeling activity is used for generating PSS values for each of the optical error contributions as well as combined estimates for each mirror and for the full telescope simulation. For the instruments that operate post adaptive optics correction, the mirror surface maps from the MACOS model are used as an input to the TMT adaptive-optics simulation models to form performance correction estimates. Finally, for the high-contrast adaptive optics imaging instruments another JPL developed model $^{9}$ for the Planet Formation Imager (PFI) uses the MACOS model exit-pupil phase maps as an input for its simulated contrast estimates. The PFI simulations have not been re-run on this latest data.

\subsection{Seeing Limited}

Table 2 shows a list of PSS values for the individual optical error sources for each OPD figure listed in section 3 at a zenith angle of 60 degrees, $\Delta \mathrm{T}$ of 4 degrees, and an $r_{0}$ of $200 \mathrm{~mm}$ at the zenith. The PSS values are calculated per Eq. 1. Currently a multiplied value for the full telescope PSS is used because we have discovered a significant Zernike mode cancelation that has occurred as a result of our random number seeding. A solution to this issue involves a Monte-Carlo simulation of the random telescope errors. This will require significantly more simulation data then has currently been accumulated at the time of the writing of this paper.

Figures 7(a)-7(b) show seeing limited PSS values over a zenith angle range of 0 to 70 degrees for each of the errors described in Section 3 at the same $\Delta \mathrm{T}$ and $r_{0}$. Due to space limitations, we have omitted figures related to the individual M2 and M3 error terms. In both cases these are dominated by the figuring error. The PSS curves for these mirrors are shown in the telescope PSS results shown in Figure 7(b). 


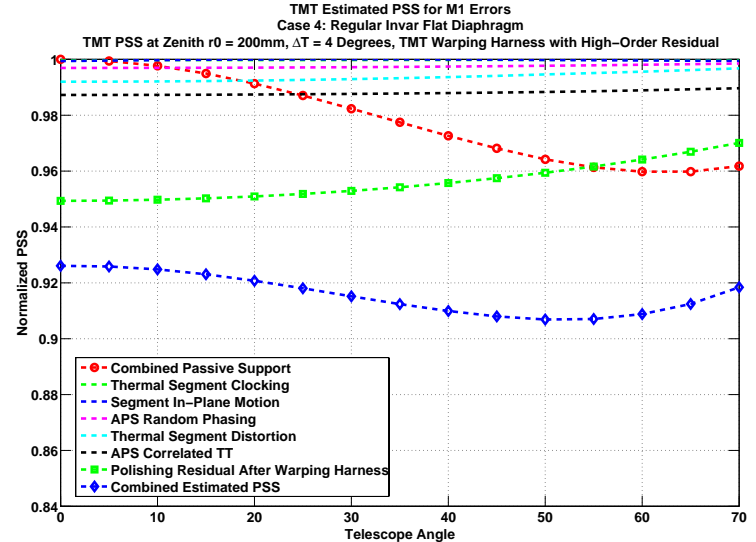

(a) M1 errors labeled in decending order at zenith pointing

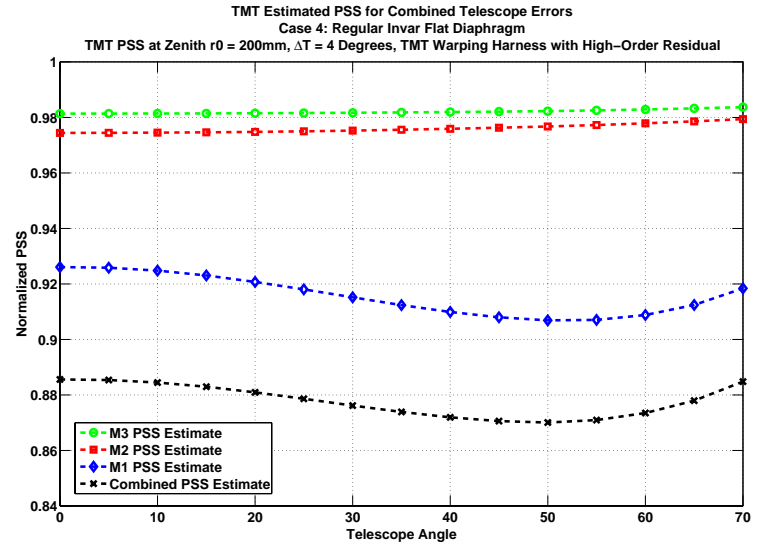

(b) Combined M1, M2, and M3 errors

Figure 7. PSS results for modeled TMT static optical errors

Table 2. Seeing Limited Normalized PSS Results: zenith angle $=60$ degrees, $\lambda=500 \mathrm{~nm}$, zenith $r_{0}=200 \mathrm{~mm}$

\begin{tabular}{|c|r|c|}
\hline & Optical Error & Normalized PSS \\
\hline \multirow{4}{*}{ M1 } & Combined Passive Support & 0.95980 \\
& APS Correlated Segment Position & 0.98891 \\
& APS Random Segment Phasing & 0.99806 \\
& Gravity Segment Clocking and Decenter & 0.99960 \\
& Warping Harness Correction & 0.96414 \\
& Thermal Segment Distortion & 0.99559 \\
& Thermal Segment Clocking & 0.99984 \\
\hline \hline \multirow{3}{*}{ M2 } & Combined M1 Errors & $\mathbf{0 . 9 0 8 8 2}$ \\
& Combined Passive Support & 0.99974 \\
& M2 Figuring Error & 0.98627 \\
& APS Position Error & 0.99071 \\
\cline { 2 - 3 } & Combined M2 Errors & $\mathbf{0 . 9 7 7 8 9}$ \\
\hline \hline \multirow{3}{*}{ M3 } & Combined Passive Support & 0.99985 \\
& M3 Figuring Error & 0.98303 \\
\cline { 2 - 3 } & Combined M3 Errors & $\mathbf{0 . 9 8 2 8 8 ^ { * }}$ \\
\hline \hline M123 & Combined Telescope Errors & $\mathbf{0 . 8 7 3 5 1}$ \\
\hline
\end{tabular}

\subsection{Adaptive Optics Corrected (NFIRAOS)}

NFIRAOS compensation of static M1/M2/M3 errors has been evaluated using TMT's in-house developed LAOS Monte Carlo simulator. ${ }^{10}$ NFIRAOS is the early-light facility AO system for TMT, ${ }^{11,12}$ and is a multi conjugate AO system that will utilize 6 laser guidestars and two deformable mirrors to provide diffraction-limited atmospheric turbulence compensation at near IR wavelengths over a 10-30" FoV. The M1/M2/M3 wavefront maps for these simulations include all the error terms described in Section 3.

A pair of LAOS simulations including and excluding the above errors were conducted to assess the quadrature difference in residual wavefront error over the IRIS $10 \mathrm{arcsec}$ FoV. All simulated wavefronts were sampled at 1/64 $\mathrm{m}$ and performance was also evaluated at that resolution. A grey pixel amplitude map sampled at 1/64 m was used to model TMT's pupil function (M1/M2/M3 obscurations). Laser Guide Star (LGS) WFSs were modeled using the polar coordinate detector array and a constrained matched filter spot position estimation algorithm, whereas the two tip/tilt (TT) and tip/tilt/focus (TTF) Natural Guide Star (NGS) WFSs were modeled geometrically as RMS best-fit tilt measurements. The NGS asterim was placed at the vertices of an equilateral triangle of 
circumradius equal to 30 arcsec at the center of the FoV. Performance has been evaluated for all modes in the LGS-controlled subspace (i.e. all residual wavefront modes orthogonal to the $2 \mathrm{TT}$ and 3 tilt anisoplanatism (TA) modes controlled using the NGS WFSs). The input static M1/M2/M3 errors had a wavefront error in the LGS-controlled subspace of $146 \mathrm{~nm}$ and a residual of $35 \mathrm{~nm}$ was found to be left uncorrected. This compares well with the $45 \mathrm{~nm}$ uncorrected error listed in the AO error budget. ${ }^{13}$

\subsection{Sensitivity Analysis}

Sensitivity analysis of parameters inherent to the errors described in Section 3 can show us how we can improve the TMT performance. We conducted two types of parameter studies, one related to the figuring error structure function specification and one related to the APS integration time. The results of these studies suggest that the M1 segment figuring error has the largest sensitivity relative to the M2 and M3 figuring error terms or that of the APS integration time.

As described in Section 3.1.4 the M1 segment figuring error is calculated using a structure function that is scaled by the atmosphere $r_{0}$. The current baseline for the M1 segments structure function $r_{0}$ value is $1.0 \mathrm{~m}$. We have run simulations varying this $r_{0}$ value as shown in Fig. 8. This sensitivity suggests that there is a large benefit of image performance with an increase in the segment figuring structure function $r_{0}$ value. The PSS reaches an asymptotic limit of $\sim 0.97$ as the residual surface error is dominated by the low-order additional allowance errors shown in Table 1.

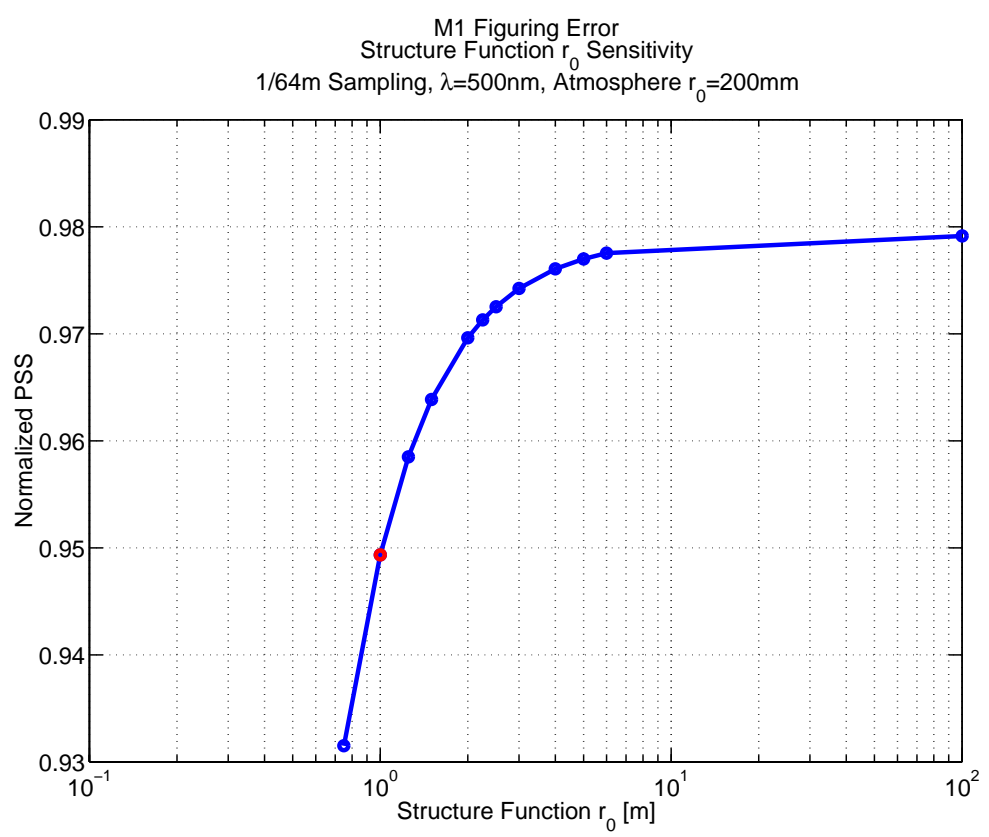

Figure 8. M1 segment figuring error $r_{0}$ sensitivity. Current baseline is $r_{0}=1.0 \mathrm{~m}$.

\section{SUMMARY}

To date we have constructed a high-resolution optical model of TMT. This model contains error terms for each of the significant contributors to static-state optical aberrations. These include passive support error, figuring error with warping harness correction, thermal disturbances, in-plane gravity effects, and segment piston, tip, and tilt errors. We have then used this model to quantify system performance in terms of a new image-plane performance metric, the normalized Point Source Sensitivity. This PSS has a convenient multiplicative feature that has been used for error budgeting and telescope performance estimation. We have generated seeing limited and AO corrected results. The PSS values for the seeing limited telescope error (at a zenith angle of 60 degrees, $\Delta \mathrm{T}$ of 4 degrees, and an $r_{0}$ of $200 \mathrm{~mm}$ at the zenith) is estimated to be 0.87351 . The LGS-controlled NFIRAOS 
corrected the input $146 \mathrm{~nm}$ RMS wavefront error to $35 \mathrm{~nm}$ RMS wavefront error. We have also conducted error term sensitivity analyses related to the mirror shape structure function parameters and APS integration time.

\section{ACKNOWLEDGMENTS}

This research was carried out in part at the Jet Propulsion Laboratory, California Institute of Technology, and was sponsored by the California Institute of Technology and the National Aeronautics and Space Administration. The authors gratefully acknowledge the support of the TMT partner institutions. They are the Association of Canadian Universities for Research in Astronomy (ACURA), the California Institute of Technology and the University of California. This work was supported as well by the Gordon and Betty Moore Foundation, the Canada Foundation for Innovation, the Ontario Ministry of Research and Innovation, the National Research Council of Canada, the Natural Sciences and Engineering Research Council of Canada, the British Columbia Knowledge Development Fund, the Association of Universities for Research in Astronomy (AURA) and the U.S. National Science Foundation.

\section{REFERENCES}

1. TMT, "TMT construction proposal," (www.tmt.org/news), 2007.

2. D. Redding et al., "MACOS manual (modeling and analysis for controlled optical systems)," NASA JPL D-9816, internal document 5, 1999.

3. B.-J. Seo, C. Nissly, G. Angeli, B. Ellerbroek, J. Nelson, N. Sigrist, and M. Troy, "Analysis of Normalized Point Source Sensitivity as a performance metric for the Thirty Meter Telescope," Proc. SPIE 7017-28, June 2008.

4. C. Nissly, M. Troy, N. Sigrist, and I. Crossfield, "TMT modeling using macos," Project Communication TMT.OPT.TEC.06.039.REL01, December 2006.

5. M. Troy, G. Chanan, S. Michaels, R. Bartos, G. Bothwell, R. Hein, M. Radin, J. Roberts, J. M. Rodgers, L. Scherr, B.-J. Seo, and D. Zimmerman, "A conceptual design for the Thirty Meter Telescope Alignment and Phasing System," Proc. SPIE, June 2008.

6. G. Chanan, M. Troy, and I. Crossfield, "Predicted measurement accuracy of the TMT Alignment and Phasing System," To be published, 2006.

7. B.-J. Seo, G. Chanan, I. Crossfield, C. Nissly, N. Sigrist, and M. Troy, "On-axis alignment performance for Alignment and Phasing System of Thirty Meter Telescope," Project Communication TMT.CTR.TEC.07.020.REL01, December 2007.

8. M. K. Cho, A. Corredor, S. Pootrakul, K. Vogiatzis, and G. Z. Angeli, "Thermal performance prediction of the TMT optics," Proc. SPIE, June 2008.

9. M. Troy, I. Crossfield, G. Chanan, P. Dumont, J. Green, and B. Macintosh, "Effects of diffraction and static wavefront errors on high-contrast imaging from the Thirty Meter Telescope," Proc. SPIE, July 2006.

10. L. Gilles, L. Wang, and B. Ellerbroek, "Modeling update for the Thirty Meter Telescope laser guide star dual-conjugate adaptive optics system," Proc. SPIE, June 2008.

11. B. Ellerbroek, S. Adkins, D. Andersen, J. Atwood, C. Boyer, P. Byrnes, R. Conan, L. Gilles, G. Herriot, P. Hickson, E. Hileman, D. Joyce, B. Leckie, M. Liang, T. Pfrommer, J.-C. Sinquin, J.-P. Véran, L. Wang, and P. Welle, "Progress towards developing the TMT adaptive optical systems and their components," Proc. SPIE, June 2008.

12. G. Herriot, D. Andersen, J. Atwood, C. Boyer, P. Byrnes, B. Ellerbroek, L. Gilles, P. Hickson, J.-P. V. B. Leckie, L. Wang, and P. Welle, "NFIRAOS - TMT's initial adaptive optics system," Proc. SPIE, June 2008.

13. T. Mast and J. Nelson, "TMT image size and wavefront error budgets," Project Communication TMT.OPT.TEC.07.021.REL01, July 2007. 